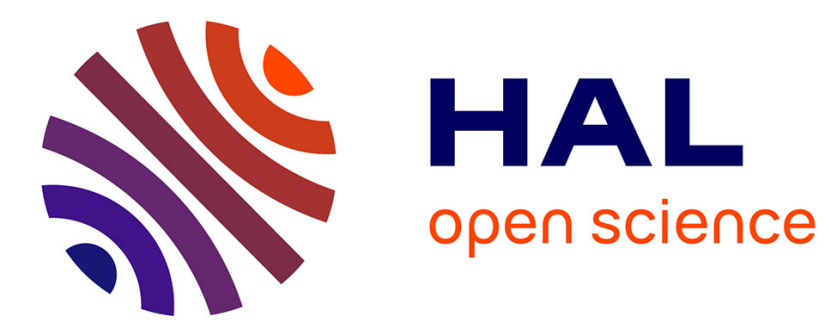

\title{
Inquisiteurs entre deux mondes
}

Jean-Paul Rehr

\section{- To cite this version:}

Jean-Paul Rehr. Inquisiteurs entre deux mondes. Raison Présente, 2019, Fanatismes, 212 (4/2019), pp.13-22. hal-02446390

\section{HAL Id: hal-02446390 \\ https://hal.science/hal-02446390}

Submitted on 24 Jan 2020

HAL is a multi-disciplinary open access archive for the deposit and dissemination of scientific research documents, whether they are published or not. The documents may come from teaching and research institutions in France or abroad, or from public or private research centers.

Copyright

L'archive ouverte pluridisciplinaire HAL, est destinée au dépôt et à la diffusion de documents scientifiques de niveau recherche, publiés ou non, émanant des établissements d'enseignement et de recherche français ou étrangers, des laboratoires publics ou privés. 


\section{INQUISITEURS ENTRE DEUX MONDES}

Jean-Paul Rehr*

[NB version final de cet article, avec numerotation, se trouve dans Raison Présente $\mathbf{n}^{\circ} 212$ $(4 / 2019)]$

\section{LA PROBLÉMATIQUE DE L'INQUISITION DANS L'HISTOIRE}

«L'Inquisition est un sujet d'une nature si particulière que quiconque tente d'en parler avec impartialité comprend qu'il a entrepris d'aborder un domaine très difficile». Ainsi commence la partie consacrée à l'Inquisition dans le livre richement illustré Cérémonies et coutumes religieuses de tous les peuples du monde, œuvre de deux protestants français, Bernard Picard et Jean Frédéric Bernard, fuyant la contre-réforme et réfugiés à Amsterdam, le livre a connu un succès international au XVIIIe siècle. Dans leur tentative de présenter « objectivement» les religions du monde, un des premiers essais pour comparer les religions, ils s'affrontent à cinq siècles d'histoire des Inquisitions catholiques. Deux cent cinquante ans plus tard, l'historien Edward Peters avoue dans son livre magistral Inquisition (1989) que la tâche n'est pas plus aisée pour l'historien moderne « tant mythe et histoire de l'Inquisition sont étroitement entrelacés. Démêler l'un de l'autre entraine à toucher quelque chose qui résonne encore dans la conscience moderne et soulève le soupçon que l'historien ne fait que créer un nouveau mythe pour remplacer l'ancien ».

L'Inquisition en est venue à représenter l'étendue sans limites du fanatisme religieux : une figure sainte qui commet des « actions mauvaises et injustes » en utilisant des «méthodes tyranniques » incluant la torture et la mise à mort. Cette image s'est incarnée dans de nombreuses formes idéologiques : elle a une origine confessionnelle, un exemple pour les protestants de tout ce qui péchait chez les « papistes » de la Sainte Église catholique et romaine. Depuis le début du XXe siècle, on l'a vue comme un problème lié au fanatisme religieux en général, qui freine le progrès de l'humanité dans la connaissance et la science. En dépit de ces polémiques, les historiens des sociétés du Moyen Âge et des débuts de la période moderne sont revenus aux sources pour éclairer les origines de l'Inquisition. C'est dans l'unité des deux sphères d'activité - la religion et la politique -, que la théorie politique occidentale a tenté de séparer depuis les Lumières, qu'on a trouvé des réponses (en clair, personne n'aurait compris la séparation de ces deux sphères avant le xiiie siècle). On voit que les premières inquisitions espagnole et portugaise qui visaient les juifs et les musulmans convertis mais suspects (et

* Université Lumière Lyon 2-CIHAM. Traduction en français par Eve Wasmuth, revue par la rédaction 
traquait les protestants) se situent à l'avant-garde des tentatives des monarchies catholiques pour pacifier leur État-Nation naissant. L'extension de ces inquisitions au Nouveau Monde (Amérique du Sud et Amérique centrale) fut une opération coloniale qui évolua rapidement d'une enquête sur l'hérésie parmi les chrétiens au renforcement de la moralité et à la surveillance de la conversion des populations autochtones. L'Inquisition romaine n'était pas faite pour extirper les « idées nouvelles », comme celles de Galilée, mais pour se défendre face au protestantisme qui menaçait l'État papal. Bien évidemment, rien de tout cela ne vise à relativiser ou à minimiser la brutalité physique, psychologique ou sociale exercée au nom de l'Inquisition. Mais si ces inquisitions sont maintenant bien comprises comme des institutions de l'Église, idéologiquement inséparables de la construction des premiers ÉtatsNations par des monarques catholiques, le terme de « fanatisme religieux » apparaît moins comme une caractérisation que comme une expression facile qui obscurcit notre compréhension.

Les historiens utilisent le terme d'« offices » pour décrire ces Inquisitions car c'était des organismes centralisés et permanents au sein de l'Église au service de leurs ambitions modernisatrices. Ce qui les a précédés au Moyen Âge étaient des commissions ad hoc créées directement par le pape. Ainsi les premières enquêtes inquisitoriales, celles du Toulousain dans les années 1230, étaient déléguées, au cas par cas, aux nouveaux ordres mendiants, Dominicains et Franciscains, et considérées comme une partie de leurs devoirs pastoraux.

Si les médiévistes s'accordent en général sur la constitution de ces toutes premières enquêtes, il n'en va pas de même sur leur origine. Le débat entre historiens ne porte pas sur les origines techniques : les premières enquêtes inquisitoriales sur la dépravation hérétique (inquisitio heretice pravitatis) ont été décidées par le pape Grégoire IX. Il s'agissait d'une modification de l'inquisitio, un type de procès de l'ancien droit romain, ressuscité au xiie siècle pour poursuivre des infractions à la loi de l'Église commises par des clercs et qui est encore la base de la plupart des systèmes juridiques de l'Europe continentale aujourd'hui. L'inquisitio heretice pravitatis a supprimé les protections de l'inquisitio romaine : les témoins sont devenus des confessants dont les propos pouvaient les conduire à s'auto-incriminer pour hérésie ; ils n'avaient pas droit au conseil d'un avocat et étaient incarcérés s'ils refusaient de témoigner (c'est-à-dire confesser et avouer, en latin, confessio indique les deux sens du mot) ; ils n'étaient pas autorisés à entendre les accusations dont ils auraient pu être l'objet, s'il y en avait.

L'historiographie nous enseigne que les premières enquêtes inquisitoriales ont été inventées pour extirper l'hérésie des Cathares, une secte de chrétiens dualistes qui croyaient, comme les anciens Manichéens, que Dieu avait créé le monde spirituel tandis que le Diable avait créé le monde matériel. Ils faisaient peser une menace existentielle sur l'Église romaine, rejetant les sacrements du baptême et 
du mariage, ainsi que la résurrection du Christ, et les remplaçant par leurs propres rituels. Au xiie siècle, on les trouvait dans toute l'Europe, de l'Allemagne à l'Italie du nord, mais ils étaient particulièrement gênants dans les terres du comte de Toulouse, Raymond VI. Là, les églises catholiques s'étaient vidées, les hérétiques ayant bâti une contre-église avec ses propres évêques et ses prêtres (les parfaits) « comme des renards dans les vignes du seigneur ». Après avoir tenté de ramener les Cathares au catholicisme par des missions de prêcheurs - dont Saint Dominique (de Guzman) qui finalement fondera l'ordre des Frères prêcheurs, les Dominicains, à Toulouse) la menace que représentaient les hérétiques du Comté de Toulouse pour l'âme chrétienne fut jugée si grave que le pape Innocent III appela à la croisade. En 1209, une armée de croisés, approuvée par le roi de France Philippe Auguste, descendit vers le sud-ouest pour mener la guerre sainte contre les hérétiques et leurs soutiens dans la noblesse. Lorsque vingt ans plus tard, en 1229, fut signé le traité de Paris entre Raymond VII et le roi de France Louis IX (Saint Louis), plusieurs rois et des milliers et des milliers de combattants et de noncombattants et d'hérétiques avaient trouvé la mort et de nombreux nobles qui avaient soutenu les Cathares (faidits) avaient été chassés de leurs terres par les nobles venus d'Île-de-France. Le traité obligeait le comte de Toulouse à extirper de ses terres les résidus de l'hérésie cathare. Après avoir échoué pendant plusieurs années à trouver les moyens de traiter l'hérésie, le pape Grégoire IX autorisa les Dominicains vers 1233 à mener des enquêtes inquisitoriales pour venir enfin à bout de la peste cathare.

Le problème pour l'étude de l'Inquisition est que les Cathares n'ont pas existé.

\section{UNE GRANDE ENQUÊTE INQUISITORIALE DE 1245 À 1246}

Parmi les manuscrits les plus précieux conservés dans les coffres de la Bibliothèque municipale de Toulouse se trouve le manuscrit Ms. 609 intitulé « Interrogatoires subis par des hérétiques albigeois par-devant frère Bernard de Caux, inquisiteur, de 1245 à 1253 ». Ce manuscrit de 254 folios contient un registre des dépositions faites à Toulouse devant les Dominicains Bernard de Caux et Jean de SaintPierre. Le manuscrit est presque entièrement composé de dépositions d'une inquisition menée entre mai 1245 et août 1246. Au cours de ces quatorze mois, plus de 5500 habitants issus de 106 villes et villages du Lauragais - la région entre Toulouse et Carcassonne - ont voyagé à pied, à cheval ou en charrette pour « faire des dépositions sur eux-mêmes et d'autres, morts ou vivants, concernant des crimes d'hérésie ». Ils représentaient trois générations, des chevaliers et des dames, des tisseurs et des tailleurs de pierres, des changeurs de monnaie et des scribes, des charbonniers et des vachers, qui évoquaient d'anciens souvenirs remontant aussi loin que les années 1180. 
C'est la plus grande enquête inquisitoriale connue de l'époque médiévale : en écrivant un historique de son ordre deux générations plus tard, l'inquisiteur dominicain Bernardo Gui (célèbre comme inquisiteur dogmatique contrairement au « détective » franciscain Guillaume de Baskerville qui apparaît dans Le Nom de la rose d'Umberto Eco), a salué en Bernard de Caux le « marteau des hérétiques » (un homme saint rempli de Dieu, continue Guillaume de Gui, dont le corps, exhumé pour déplacement vers son lieu de repos éternel des décennies après sa mort, fut retrouvé parfaitement préservé). Le registre reproduit dans le Ms. 609 est une copie des volumes quatre et cinq d'un ensemble d'au moins dix volumes originaux (selon les notes en marge du copiste). Il a été créé pour les inquisiteurs de Narbonne quelques dix années plus tard et au cours des siècles, il est passé dans les archives des Dominicains à Toulouse et enfin dans la collection des manuscrits de Toulouse. La théorie des Cathares dualistes paneuropéens, et leur rôle en tant que raison d'être des premières enquêtes inquisitoriales, ont été étudiés et exposés par le théologien strasbourgeois Charles Schmidt dans son Histoire et doctrine de la secte des Cathares ou Albigeois (1848). Schmidt s'est arrêté sur le nom « Cathare » rencontré dans les rapports d'hérésie découverts à Cologne car il croyait, comme beaucoup d'historiens le croient toujours, que les hérétiques de Rhénanie dans les années 1140 étaient les mêmes que ceux qui ont provoqué la croisade des Albigeois 60 ans plus tard. La théorie était déjà vieille d'une génération et établie comme un fait historique quand le Ms. 609 fut catalogué comme preuve de l'existence des Cathares. Selon l'influent Célestin Douais, abbé et professeur de religion à l'Institut Catholique de Toulouse, le registre rendait compte d'une « enquête sur l'état religieux du pays » prouvant l'existence des Cathares et de leurs hérésies. L’opinion de Douais et les méthodes de recherches qui l'ont formée il y a 130 ans ont été adoptées par la plupart des grands historiens de la « grande hérésie » du Moyen Âge.

Cela ne signifie pas que le catharisme a été largement étudié par des historiens qualifiés. L'étude des Cathares a été, jusqu'à il y a environ cinquante ans, le domaine de spécialistes de la religion et de la théologie chrétienne, fréquemment des clercs comme Douais, leurs étudiants, ou ceux qu'intéressaient la nature exacte et les origines du dualisme cathare. Dans les années 1960, l'hérésie médiévale faisait déjà partie des recherches historiographiques portant sur la « vie quotidienne » des « gens ordinaires ». Ces recherches portent la marque de l'influence exercée après-guerre par la grande école d'histoire française des Annales (illustrée par Fernand Braudel, Georges Duby, Jacques Le Goff). Elles rencontraient l'intérêt du public, entretenu par des oeuvres telles que Le Bûcher de Montségur (1959) de Zoé Oldenbourg et le docudrame de cinq heures « Les Cathares - La Croisade et L’Inquisition » (1966) diffusé par la télévision nationale française. Cet intérêt pour les Cathares continue d'être alimenté par les ouvrages des érudits locaux du sud-ouest, comme l'avocat Jean 
Duvernoy et le journaliste Michel Roquebert.

Le Ms. 609 et la poignée d'autres registres d'inquisitions sont exceptionnels par le fait qu'ils sont l'unique témoignage direct de « la vie quotidienne » de l'époque médiévale jusqu'à la fin du treizième siècle ; les historiens médiévistes dépendent en grande partie des chroniques, des chartes et de la législation ecclésiastique et de toute autre documentation juridique (des monarques, de la noblesse et de l'Église). Dans les années 1980, les successeurs des fondateurs des Annales ont commencé à mettre en place les méthodes pour interroger ces sources médiévales profondément partiales et étudier les mentalités et la vie en société. ${ }^{1}$ Monique Zerner, une élève de Georges Duby, et des historiens tels que Jacques Chiffoleau et JeanLouis Biget, ont reconnu qu'ils posaient les mêmes questions sur les sources de notre compréhension de l'hérésie médiévale. Les ouvrages collectifs dirigés par Monique Zerner Inventer l'hérésie ? Discours polémiques et pouvoirs avant l'Inquisition (1998) et L'Histoire du Catharisme en discussion : le 'Concile' de Saint-Félix, 1167 (2001), ont établi plusieurs faits. Le premier a montré que les sources de notre approche de l'hérésie « cathare » sur les terres du comte de Toulouse avant la croisade albigeoise ne pouvaient être considérées comme des rapports objectifs sur l’hérésie. De plus, ces chercheurs ont démontré le rôle clé joué par l'ordre cistercien dans la diabolisation, à l'œuvre depuis plus d'un siècle, d'une hérésie supposée et dans la diabolisation des nobles de Carcassonne jusqu'à Toulouse, accusés de l'avoir soutenue et protégée. Le dernier travail a mis en cause l'authenticité d'un document essentiel pour justifier l'existence d'une Église cathare liée aux hérétiques dans les Balkans; il est maintenant considéré par beaucoup comme étant un faux ${ }^{2}$. Tandis que Zerner et ses collègues menaient leurs investigations sur les polémiques anti-hérésie avant les Croisades et les premières enquêtes inquisitoriales, Mark Gregory Pegg de l'université de Princeton menait une recherche sur les dépositions dans Ms. 609. ${ }^{3}$ Bien qu'ils aient opéré sans se connaître, ils partageaient une méthodologie similaire. Pegg a écarté les polémiques ecclésiastiques et a analysé les dépositions en tant qu'historien anthropologue. Ses vues, qui semblent aujourd'hui évidentes, étaient radicales. Un passage typique des dépositions comme celle d'Arnaud Garnier du MasSaintes-Puelles, le premier du manuscrit, permet de les illustrer. Il raconte un événement (toujours enregistré à la troisième personne) :

« Item. Il a vu, à un endroit dit « Olivier », Bernard de Mairevila et son compagnon, hérétiques. Il a vu avec lesdits hérétiques, Garnier, le père du témoin ; Guilhem Vital ; Guilhem Barbas ; Jordanet del Mas

1 Montaillou, village occitan de 1284 à 1324 d'Emmanuel Le Roy Ladurie (Gallimard, 1975) était un produit direct des méthodologies des Annales.

2 R.I. Moore (2017), Hérétiques : résistances et répression dans l'Occident médiéval, Paris, Belin.

3 Mark Gregory Pegg (2001), The Corruption of Angels: The Great Inquisition of 1245-1246, Princeton, Princeton University Press. Traduction en français par Julien Théry, sous presse. 
; et plusieurs autres dont il ne s'est pas souvenu. Le témoin et tous les autres ont adoré lesdits hérétiques là-bas. C'était il y a environ sept ans. »

Après avoir énuméré quelques événements, la déposition, comme presque tous les aveux, se termine par un résumé de « croyances » hérétiques :

« Il a dit qu'il croyait que les hérétiques étaient de bons hommes et avaient une bonne foi et étaient vrais et amis de Dieu. Il a entendu les hérétiques dire que Dieu n'avait pas fait des choses visibles, mais le témoin ne croyait pas en cette erreur. Il n'avait pas entendu les hérétiques parler du baptême, de l'hostie consacrée, du mariage, de la résurrection de la chair. »

La première observation, qui est aujourd'hui une évidence, est que le mot « Cathare » n'est mentionné nulle part dans les 5500 dépositions de personnes qui étaient supposées vivre dans la « citadelle de l'hérésie » du Lauragais (et le terme « Cathare » n'apparait dans aucun document du Midi de l'époque). Mais surtout, il n'y a aucune mention de parfaits, ni aucun indice à leur sujet. Ce que nous pouvons observer c'est une liste en constante évolution d'hommes et de femmes, les bons hommes (boni homines) et bonnes femmes (bonae feminae), allant des enfants aux personnes âgées, qui bénéficiaient parfois d'une image de saints dans leur communauté. Les rôles des adultes variaient: conseillers, médiateurs, prononçant des sortes de prêche ou effectuant certains rituels auprès des mourants. Malgré les nombreuses références à des « prédicateurs d’hérésie errants », la plupart des bons hommes étaient restés dans leurs villages jusqu'à ce que les premières enquêtes inquisitoriales les eussent forcés à vivre en nomades à la recherche d'abri et de protection.

Une lecture attentive des dépositions en fonction des dates des souvenirs montre que toute « structure » explicite dans laquelle les bons hommes ont été classés, avec, au mieux, peu de traces, est fragilisée par les effets de la guerre et de l'inquisition. Il en va de même pour le contenu de ce que les bons hommes ont prêché : seuls les souvenirs des années 1230 commencent à montrer qu'on a entendu certains bons hommes parler des sacrements - baptême, mariage, résurrection - et du dualisme. Un certain nombre de dépositions montrent que la seule fois où le témoin a entendu discuter du dualisme, c'était par les prêtres catholiques eux-mêmes. Ce qui ressort des dépositions, comme celles d'Arnaud Garnier, où les inquisiteurs ont certainement insisté pour obtenir des preuves d'hérésie, c'est qu'il n'y avait aucune cohérence dans le message des bons hommes à propos des sacrements. Il se peut très bien que les seuls qui se souciaient des sacrements étaient les inquisiteurs. Il faut rappeler que c'est à peine une génération plus tôt que le pape Innocent III avait convoqué le quatrième Concile du Latran qui a 
mis en place de nombreux canons du sacerdoce catholique moderne (de nombreux villages n'avaient même pas leur propre prêtre), de leur instruction formelle à leur contrôle moral, et contraint la population profane à suivre les sacrements, y compris à se confesser au moins une fois par an.

Le problème identifié par Pegg est le suivant : les historiens des religions ont construit des Cathares à partir de bons hommes et de bonnes femmes en se basant sur une lecture sélective de preuves inquisitoriales généralement dérivées a) d'auteurs ecclésiastiques hostiles et b) façonnées par les représentations des théories savantes du XIXe siècle portant sur la manière dont une religion devrait être structurée. Le témoignage le plus proche de l'expérience vécue, les registres inquisitoriaux, doit donc être lu avec soin pour séparer ce qui a trait à l'existence réelle des témoins de ce que les inquisiteurs faisaient quand ils posaient leurs questions et enregistraient leurs réponses en termes d'hérésies. Pour Pegg, cela devient doublement difficile, car « ce qui a transformé ces individus en hérétiques, ce qui a transformé l'accusation en réalité, c'est la violence de la croisade des Albigeois et la persécution des premiers inquisiteurs ».

\section{UNE NOUVELLE APPROCHE DE L'ENQUÊTE INQUISITORIALE DE 1245-1246}

Ce qui précède constitue le contexte dans lequel s'inscrit ma propre recherche sur Ms 609 et « la grande enquête inquisitoriale ». Il serait compréhensible de se sentir perdu dans tous ces arguments. Le « débat cathare ${ }^{4}$ lui-même souffre d'un problème de méthode, enraciné, comme nous l'avons vu, dans la lecture sélective des sources. Cela n'est nulle part plus évident que dans les études utilisant Ms 609 - la plupart des études qui y ont fait référence s'appuyaient sur une transcription fragmentaire, rarement sur le manuscrit proprement dit (Pegg étant l'exception notable). Le résultat est un biais de sélection profondément enraciné qui agit comme une boucle de rétroaction : on trouve dans le registre précisément ce que l'on cherchait, puis on déclare que c'est tout ce qu'il contient ${ }^{5}$. C'est pourquoi je me suis lancé dans l'édition de Ms 609 dans son intégralité en édition numérique (hébergée par le CNRS à l'adresse http://medieval-inquisition.huma-num.fr/). Une édition numérique permet de traiter le registre à la fois comme un texte et une base de données. Tous les aspects du contenu de chaque déposition ont été codés pour des raisons de transparence : personnes et rapports, lieux, dates, « actes hérétiques » et événements sont codés et reliés de manière à permettre l'enquête sur les dépositions sous différents angles. Cette méthode révèle efficacement les habitudes de travail des inquisiteurs

4 Voir Antonio Sennis, éd. (2016), Cathars in Question, Suffolk, Boydell \& Brewer ; et Jean-Louis Biget, éd., Le « catharisme » en questions, Cahiers de Fanjeaux, 55, sous presse.

5 Jean-Paul Rehr (2019), Re-Mapping the "Great Inquisition" of 1245-46: The Case of Mas-Saintes-Puelles and SaintMartin-Lalande, Online Journal of Humanities, 5(1): 1-53. 
Bernard de Caux et Jean de Saint-Pierre.

Les formulaires des premiers inquisiteurs deviennent limpides après la lecture de quelques dépositions que chaque historien commente : chaque déposition est composée d' « événements » individuels, pratiquement tous structurés selon le même schéma. En revenant sur la confession d'Arnaud Garnier mentionnée ci-dessus, nous trouvons : un lieu où un événement hérétique s'est produit (Olivier) ; les acteurs « hérétiques » et les autres personnes impliquées (y compris le témoin); l'action hérétique (adorer); et une date. Les historiens ont été prompts à commenter la maigre qualité, sèche et répétitive, des formulaires et en ont proposé des explications. Certains se sont demandé si les inquisiteurs étaient simplement indifférents aux détails ; d'autres considéraient ces formulaires comme une évidence de l'hérésie cathare ; et d'autres encore attribuaient leur existence à une nécessité pratique : les inquisiteurs ont dû traiter des milliers de témoins, une charge de travail qui ne pouvait être gérée que par la réduction efficace des dépositions en check-lists. Plusieurs historiens ont fait remarquer que les deux inquisiteurs laissaient beaucoup de pistes inexplorées - des personnes associées à bons hommes aux « hérésies » qui n'apparaissent qu'une seule fois - un coût supplémentaire de la vitesse avec laquelle les inquisiteurs devaient opérer.

Après avoir encodé et mis en relation les personnes, les événements et les dates des « événements » individuels, c'est-à-dire examiné l'enquête inquisitoriale dans son intégralité, une tendance se dégage : a) les mêmes récits se répètent dans les dépositions incriminant b) de petits groupes de personnes dans chaque village. Comprendre qui sont ces groupes de personnes nous oblige à reconstruire le contexte, la vie vécue par les villageois incriminés dans la déposition - ce détail ne se trouve pas dans les polémiques cisterciennes, ni dans les traités anti-hérésie écrits par les scolastiques de l'époque. Les historiens ne se sont intéressés qu'à une célèbre seigneurie figurant dans le registre, affirmant que l'attention des inquisiteurs était centrée sur elle en raison de la fameuse adhésion des nobles aux Cathares. En fait, la noblesse tombe au deuxième, voire au troisième rang, dans l'intérêt des inquisiteurs ; leur place dans l'historiographie est la création d'historiens qui ont choisi de placer la noblesse au cœur d'un récit cathare (ce qui convient parfaitement au marketing des « châteaux cathares $\gg)$.

Nous pouvons nous servir ici de documents peu utilisés par les historiens de l'hérésie, mais qui sont précieux pour les données sociales qu'ils contiennent : les chartes et les serments. Dans le Trésor des chartes des Archives nationales à Paris, on trouve une série de serments prêtés dans des villages du Lauragais en 1243, juste après l'échec de la rébellion du comte Raymond VII et d'autres nobles contre Louis IX. Ces serments étaient prêtés à Louis IX dans chaque village par des nobles, des consuls et des 
bons hommes. Ils jurèrent de mettre en garde leur comte Raymond contre toute nouvelle rébellion, de rester fidèles au roi dans la guerre contre leur comte et de soutenir l'Église contre l'hérésie. Les noms des consuls de villages dans ces serments (les consuls dont les sceaux ont validé les serments !) sont ceux des familles retrouvées au centre des accusations d'hérésie dans les villages du Lauragais.

Lorsque les dépositions sont ré-examinées, il devient évident que les incriminations des consuls ne sont pas le résultat de leur adhésion personnelle à l'hérésie. Les aveux d'Arnaud Garnier, liés à ceux des autres habitants du village, reflètent l'orientation des inquisiteurs qui recherchent des informations spécifiques sur des personnes très spécifiques selon des critères qui vont au-delà de l'adhésion personnelle à « l'hérésie », clairement centrés sur les familles consulaires. Ce critère permettait aux inquisiteurs de passer à côté de nombreux noms évidemment liés à «l'hérésie ». Ce critère est la raison pour laquelle la plus grande inquisition connue du Moyen Âge a pu être exécutée aussi rapidement sur un territoire aussi vaste.

Il reste encore beaucoup à faire pour comprendre « la grande enquête inquisitoriale » de 12451246, en s'inspirant des mythes des Cathares. Quel rôle ont joué les consuls de village qui en ont fait le sujet des inquisiteurs, ces mêmes consuls qui avaient prêté serment deux ans auparavant à Louis IX, le défenseur capétien de la foi chrétienne, et contre leur seigneur, le comte de Toulouse ? Comment devons-nous comprendre l'apparition des bons hommes, les « hérétiques » des dépositions, sur les serments susmentionnés pour lutter contre l'hérésie ? La plupart des récits dans les dépositions datent de 5 à 15 ans auparavant et, selon les dépositions, leurs auteurs s'étaient déjà confessés à d'autres inquisiteurs et avaient été « pardonnés », que recherchaient les inquisiteurs? Enfin, après des années de résistance, pourquoi Raymond VII a-t-il finalement accepté cette vaste enquête inquisitoriale sur ses terres qui semble avoir visé les consuls ? Ces questions nous ramènent encore à cette frontière artificielle moderne entre religion et politique. Malgré la croyance réelle de ces inquisiteurs et leur intérêt déclaré pour le salut des âmes individuelles et leur préparation à la rencontre d'un Dieu invisible, ils ont clairement agi de concert avec le monde matériel visible, et l'ont remodelé. 